\section{Preditores do retardo de crescimento linear em pré-escolares: uma abordagem multinível}

\author{
Determinants of linear growth retardation in \\ Brazilian preschool children: a multilevel approach
}

\author{
${ }^{1}$ Escola de Nutrição, \\ Universidade Federal da \\ Bahia, Salvador, Brasil. \\ 2 Instituto de Saúde Coletiva, \\ Universidade Federal da \\ Bahia, Salvador, Brasil. \\ ${ }^{3}$ Instituto de Matemática, \\ Universidade Federal da \\ Bahia, Salvador, Brasil. \\ 4 Núcleo de Estudos \\ Epidemiológicos em Nutrição \\ e Saúde, Universidade de \\ São Paulo, São Paulo, Brasil. \\ 5 Instituto de Saúde, \\ Secretaria Estadual de Saúde \\ de São Paulo, São Paulo, \\ Brasil.
}

Correspondência L. P. M. Oliveira Escola de Nutrição, Universidade Federal da Bahia.

Rua Araújo Pinho 32, Salvador, $B A$ 40110-150, Brasil. lucipmo@ufba.br

\section{Abstract}

This study focuses on the determinants of linear growth deficit in under-five children using multilevel modeling. The sample included 3,746 preschoolers from 15 Brazilian municipalities (ten from the State of Bahia and five from São Paulo), with a three-tier analysis (municipalities, households, and children). Municipal data were obtained from the 1991 National Census. Individual and household data were collected from 1999 to 2001 using structured questionnaires and measuring children's weight and height. Analysis used the MLwiN software. In the final multilevel model, low HDI and prenatal care at the municipal level, poor environmental and economic conditions and maternal schooling at the household level, and low birth weight, incomplete immunization, prior history of malnutrition, and no report of breastfeeding at the individual level were strongly associated with linear growth deficit. Total variability of deficit explained by the model was $27.4 \%$, of which $51.3 \%, 33 \%$, and $15.7 \%$ were explained, respectively, by individual, household, and municipal variables.

Growth and Development; Malnutrition; Preschool Child
Lucivalda P. M. Oliveira 1

Mauricio L. Barreto 2

Ana Marlúcia O. Assis 1

Antonio C. R. Braga-Junior ${ }^{3}$

Maria F. F. Pussik Nunes 2

Nelson F. Oliveira ${ }^{3}$

Maria Helena D'Aquino Benício 4

Sônia Isoyama Venâncio 5

Sílvia Regina D. Médici Saldiva 5

Maria Mercedes Loureiro Escuder 5

\section{Introdução}

A desnutrição na infância, expressa pelo comprometimento do crescimento linear e/ou ponderal, é ainda um dos principais problemas de saúde enfrentados pelos países em desenvolvimento, quer pela elevada prevalência 1,2 , quer pela carga de morbidade que se associa a esse evento 3,4 ; embora, tenha sido registrada tendência de declínio da prevalência da desnutrição na maioria destes países a partir da década de 70. Ressalta-se ainda que, na atualidade, em muitas regiões do mundo, em especial na África e Ásia, foi observada desaceleração na queda dessa prevalência 5 . A tendência de declínio da desnutrição na infância é também registrada no Brasil, que se iniciou na década de 70, sobretudo nas áreas urbanas 6 de todas as regiões geográficas do país.

No entanto, o Nordeste brasileiro, uma das regiões mais pobres do país, ainda que tenha experimentado as mais elevadas taxas de declínio da desnutrição, ainda continua a exibir as mais altas prevalências de déficits antropométricos (<-2DP do padrão NCHS). Informações oriundas da Pesquisa Nacional sobre Demografia e Saúde (PNDS) realizada em 1996, indicavam déficits mais elevados tanto para o indicador altura/idade $(17,9 \%)$ quanto para peso/idade $(8,3 \%)$, quando comparados com aqueles identificados para a Região Sul (respectivamente de 5,1\% e 2\%), uma das mais ricas do país 7. Salienta-se também que essas prevalências eram ainda mais elevadas pa- 
ra o Nordeste rural, cuja prevalência do déficit altura/idade foi de $19 \%$ e peso/idade de $9,2 \% 7$.

Dados recentes (2001-2003) do Ministério da Saúde, oriundos de crianças menores de sete anos de idade de municípios do Nordeste do Brasil, resultantes da avaliação do Programa Bolsa Alimentação, indicam a tendência atual da manutenção das altas prevalências de déficit no crescimento linear $(15,1 \%)$ e ponderal $(10,7 \%)$ e sustentam também o diferencial da distribuição dos déficits do crescimento na infância entre as regiões 8 .

Assim, apesar da evolução favorável dos indicadores de saúde e nutrição na infância observados no Brasil, principalmente no que diz respeito às taxas de imunizações, do uso de terapia de reidratação oral e do aleitamento materno, a prevalência da desnutrição em algumas áreas geográficas do país não acompanha essa tendência e mostra as grandes disparidades na distribuição entre áreas urbanas e rurais, entre as regiões e municípios de um mesmo Estado 6 .

Seguramente as condições que ilustram essa assimetria estão fortemente vinculadas às desigualdades social e econômica e à iniqüidade em saúde observadas entre essas regiões 9 . Nesse sentido, as evidências indicam que os determinantes dos déficits antropométricos na infância configuram uma estrutura complexa e variada, que envolve múltiplos fatores do ambiente econômico, social e familiar da criança 10,11.

Nesse contexto a privação das satisfações básicas de vida imposta pela persistência das condições inadequadas do ambiente social e econômico se traduz em restrições no consumo alimentar, nas precárias condições do ambiente sanitário e na elevada carga de morbidade, promovendo o constrangimento do crescimento na infância.

O crescimento linear é um dos eventos que expressa de forma contundente o impacto cumulativo das condições do ambiente social e econômico sobre a saúde e nutrição na infância 12,13.

A busca de um modelo conceitual que melhor traduza a complexidade da determinação da desnutrição na infância tem constituído em preocupação de vários organismos. Nesse sentido, é sugerido pelo Fundo das Nações Unidas para a Infância (UNICEF) 11 um modelo, que leva em conta os diferentes níveis hierárquicos de determinação da desnutrição na infância. Nesse modelo os determinantes são alocados em diferentes níveis de hierarquia: básicos, intermediários/subjacentes e imediatos. Os determinantes básicos situam-se na esfera da produção e da distribuição da riqueza produzida na sociedade. Nesse contexto, sobressai-se o poder explicativo expresso pela pobreza e pela desigualdade econômica. As causas básicas por sua vez intermediam os fatores subjacentes, imprimindo diferenciados acessos aos bens e serviços essenciais para assegurar a subsistência, a exemplo do acesso ao alimento, ao serviço de saúde, ao saneamento e à educação. Os determinantes imediatos expressam inter-relação entre as causas básicas e intermediárias e envolvem o consumo dietético e o padrão de morbidade da criança, culminando na determinação do perfil nutricional na infância.

Embora essa abordagem proposta pelo UNICEF contemple os diferentes níveis hierárquicos dos determinantes da desnutrição, a análise estatística deve levar em consideração os diferentes gradientes de agregação destas variáveis. Nesse sentido, o modelo de análise multinível é o indicado por contemplar as condições de dependência entre os diferentes níveis e dentro dos próprios níveis explicativos do evento 14. Essa técnica, comumente usada em estudos no campo da educação, sociologia e demografia, teve o seu uso recentemente ampliado para a investigação dos eventos relacionados à saúde, que são conhecidamente multifacetados.

Assim, este estudo tem como propósito identificar variáveis preditoras do crescimento linear em crianças menores de cinco anos de municípios da Bahia e São Paulo, utilizando-se do modelo de análise multinível, podendo assim contribuir para o entendimento de um problema complexo e mesmo para a definição de ações e políticas públicas voltadas para o controle e prevenção da desnutrição na infância.

\section{População e método}

Os dados deste estudo são oriundos de uma amostra de 2.733 pré-escolares de dez municípios do Estado da Bahia e de 1.013 pré-escolares de cinco municípios do Estado de São Paulo, totalizando 3.746 menores de cinco anos provenientes dos 15 municípios estudados. Os inquéritos foram realizados no período de março de 1999 a março de 2000, no Estado da Bahia, e em novembro e dezembro de 2001 nos municípios do Estado de São Paulo.

\section{Tamanho da amostra}

A amostra deste estudo foi construída em três estágios, contemplando os diferentes níveis de hierarquia representados pela criança, domicílio e município.

O número de municípios de cada Estado incluído na amostra obedeceu ao critério da viabilidade do trabalho e à disponibilidade de recur- 
sos. Assim, decidiu-se investigar dez municípios na Bahia e cinco em São Paulo.

Para obter o número de domicílios a ser incluído na amostra, levou-se em conta a estimativa do número médio de crianças por domicílio em cada município e zona de residência (rural/ urbana), fornecida pelo Censo Demográfico de 1991 15, sendo estimados 1.887 domicílios no Estado da Bahia e 801 em São Paulo.

O cálculo do número de crianças a ser incluído na amostra de cada estado, obedeceu a critérios comuns. Para a Bahia esse cálculo foi baseado na estimativa da prevalência do déficit do indicador peso/idade $(8,3 \%)$ e da altura/idade $(17,9 \%)$ da Região Nordeste fornecida pela PNDS 7 e para o Estado de São Paulo utilizou-se a prevalência da desnutrição infantil para cada município estimada a partir de modelos preditivos de Benício \& Monteiro 16. Como era de interesse incluir na amostra as crianças residentes na zona rural e na zona urbana, optou-se por uma amostra aleatória estratificada, com alocação proporcional segundo a área de residência das crianças.

Com base nessa estratégia, foi calculado o número de criança a ser sorteado em cada município, segundo a zona de residência, resultando para a Bahia uma amostra de 2.648 crianças menores de cinco anos de idade, sendo estudadas efetivamente 2.733 crianças das áreas urbana e rural. E em São Paulo o número amostral foi de 909, sendo estudadas 1.013 crianças das áreas rural e urbana 17 .

\section{Seleção dos municípios, dos} domicílios e das crianças

Para o Estado da Bahia, a seleção dos municípios pautou-se no pressuposto a representação das diferentes áreas geográficas, com o objetivo de contemplar as diversidades sociais e econômicas. Por questões logísticas que tornavam mais factíveis operacionalizar o estudo, optou-se por selecionar os municípios dentre aqueles de 10 mil a 15 mil habitantes, e que possuíssem maiores taxas de urbanização e pequena extensão territorial $\left(<500 \mathrm{~km}^{2}\right)$; por razões semelhantes para o Estado de São Paulo, o município deveria contar com menos de 10 mil habitantes, contemplando-se, no entanto, os diferentes índices de desenvolvimento social segundo o IPRS (Índice Paulista de Responsabilidade Social), elaborado pela Fundação Sistema Estadual de Análise de Dados (Fundação SEADE) 18. De posse da listagem dos municípios de ambos os estados que atendessem a esses critérios, procedeu-se ao sorteio dessas unidades.

A seleção dos domicílios pesquisados na zona urbana de cada município foi processada por amostragem sistemática. Para a seleção dos domicílios na área rural, no Estado da Bahia identificou-se o agrupamento dos domicílios, com base nas fichas de coletas da Contagem Populacional de 1996, disponibilizado pelo escritório do Instituto Brasileiro de Geografia e Estatística (IBGE) em Salvador. Com base nesses critérios, nos domicílios visitados que contavam com crianças menores de cinco anos de idade, todas foram avaliadas e incluídas na amostra. No Estado de São Paulo optou-se por abordar todos os conglomerados rurais registrados na prefeitura local e entrevistar todas as crianças residentes no domicilio, sendo excluídos deste sorteio os domicílios rurais isolados 17 .

\section{Coleta de dados}

Os dados referentes ao município foram obtidos do Censo Demográfico de 1991 15. As demais informações utilizadas no estudo têm caráter primário. Aquelas referentes às condições sócioeconômicas, saúde materna e da criança foram obtidas utilizando-se questionários estruturados, aplicados às mães ou responsáveis pela criança. As medidas do peso e comprimento/altura foram tomadas em duplicata. Para a medição do peso utilizou-se balança microeletrônica, marca Fillizola (modelo E-150/3P), com precisão de 100g. O comprimento das crianças menores de dois anos de idade foi medido com infantômetro de madeira, especialmente construído para este fim, e a altura daquelas com dois anos ou mais foi medida por meio de estadiômetro marca Leicester Height Measure, ambos com precisão de $0,1 \mathrm{~cm}$. A padronização das medidas foi realizada de acordo com as recomendações técnicas 19 . Os dados referentes à data de nascimento foram comprovados por meio da certidão de nascimento. Todos os dados foram coletados por nutricionistas treinadas para este fim.

\section{Definição das variáveis}

A variável dependente deste estudo é representada pelo indicador antropométrico altura/idade na forma contínua, expressa em z score ${ }^{20}$. As variáveis independentes foram definidas, com base em revisão de literatura, entre aquelas que se mostraram mais consistentemente associadas ao déficit de crescimento linear. Assim, foram incluídas na análise exploratória variáveis relacionadas com a criança, tais como: sexo (masculino $=0$; feminino $=1)$, idade $(0$ a $6 ; 12$ a $14 ; 24$ a 36 ; > 36; a idade de 0 a 6 meses foi tomada como referência e as demais faixas etárias representam riscos crescentes), peso ao nascer $(\geq 2.500 \mathrm{~g}=0$; $<2.500 \mathrm{~g}=1$ ), esquema vacinal (completo $=0$; in- 
completo $=1$ ), aleitamento materno (se a criança foi alimentada ao peito independentemente do tempo ou regime de aleitamento/ $\operatorname{sim}=0$ e não = 1), percepção materna sobre a saúde da criança (muito boa $/$ boa $=0$; regular $/$ ruim $=1$ ), história de desnutrição pregressa (não = 0 e sim $=1$ ), internamento prévio (não $=0$ e $\operatorname{sim}=1$ ), morbidades referidas nos últimos 15 dias, como: diarréia, febre, coriza, perda de apetite (ausência de qualquer condição $=0$; presença de qualquer uma delas $=1$ ); essas variáveis integraram o primeiro nível da hierarquia.

As variáveis incluídas no segundo nível da hierarquia foram representadas pelas condições do ambiente sanitário do domicílio e do peridomicílio, na forma do índice ambiental, adaptado do modelo proposto por Issler \& Giugliani 21, assim construído: esgoto próximo do domicílio (não $=0$ e sim $=1$ ), destino do lixo doméstico [coleta pública/queima (0) e joga em área aberta, córrego ou rio (1)], abastecimento de água [adequado, originário da rede geral de abastecimento (0); inadequado, não originária da rede geral de abastecimento (1)], condições do sanitário [individual com descarga (0); inadequado, se não possuía, não era individual ou não tinha descarga (1)], material predominante na cobertura e parede do domicílio [bloco de tijolo (0); adobe, papelão, tábua e material reaproveitado (1)], material predominante no piso [cerâmica, cimento, tijolo (0) e terra batida (1)], condição de posse do domicílio [próprio (0), cedido, alugado, invasão, em financiamento (1)] e número de habitantes por dormitório [> 2 (0) e $\leq 2$ (4)].

Após a codificação das variáveis atribuiu-se pontuação a cada situação; a mais desfavorável recebeu valor 0 e a mais favorável recebeu pontuação 4. O somatório desses valores caracteriza as condições ambientais do domicílio e do peridomicílio e, essa variável integrou o modelo na forma contínua.

Ainda no segundo nível da hierarquia, foram incluídos: a renda per capita familiar em salário mínimo, a escolaridade materna (em anos de estudo), ambas na forma contínua, e o índice econômico, que reflete a condição econômica da família. A construção desse índice baseou-se no método proposto pela Associação Brasileira de Empresas de Pesquisa (ABEP) 22 e é utilizado também pelo IBGE no Censo Demográfico. Esse índice foi composto com base na posse dos seguintes bens: televisão em cores e/ou preto e branco, vídeo cassete, geladeira, rádio, máquina de lavar roupa e automóvel. Excluíram-se as condições de contratação de empregada mensalista, posse de aspirador de pó e freezer, originalmente mantidos na proposta da ABEP e IBGE e, por questões operacionais, não se utilizou o esque- ma de pontuação proposto por estes órgãos, que leva em conta o crescente número de cada bem existente no domicílio. Neste estudo, essas variáveis tiveram caráter dicotômico e assumiram valor 4 (para presença de pelo menos um bem no domicilio) ou 0 (ausência do bem no domicílio) e o somatório destes valores representou o índice econômico que foi usado no modelo na forma contínua.

No terceiro nível da hierarquia foram incluídas as variáveis municipais: razão de concentração de renda, razão de concentração de renda segundo área dos imóveis rurais, grau de urbanização, volume total de água consumido $\left(\mathrm{m}^{3}\right)$, arrecadação total, arrecadação per capita, renda média dos chefes de família, valor da produção animal, assistência pré-natal prestada pelo município (percentual de recém-nascidos cujas mães fizeram seis ou mais consultas no pré-natal), e Índice de Desenvolvimento Humano (IDH), este índice varia de 0 a 1 e engloba três variáveis: longevidade da população, calculada pela expectativa de vida ao nascer; escolaridade, avaliada pelo índice de alfabetização e pela taxa combinada de matrícula dos três níveis de ensino; e renda per capita em dólar, mensurada pelo PIB (Produto Interno Bruto), para manter a paridade do poder de compra entre países 15 . Todas essas variáveis do nível 3 foram acrescentadas ao modelo na forma contínua.

\section{Análise estatística}

A estrutura de dados deste estudo envolve múltiplas observações das crianças, que estão aninhadas nos domicílios, e cada domicílio está também aninhado no município pesquisado. Tal estrutura é tipicamente hierárquica, em três níveis: criança, domicílio e município. Assim, na análise estatística, adotou-se a abordagem multinível, que além de atender às necessidades específicas do desenho amostral contribui para o entendimento dos fatores determinantes da desnutrição segundo a relação hierárquica e os seus níveis de inter-relações 23,24.

Como estratégia de análise estatística, optouse pelo modelo de regressão linear ${ }^{25}$, com um intercepto aleatório, pois além de identificar a variabilidade atribuível a cada nível na determinação do déficit linear, também lida com o efeito de cluster, ou seja, a não independência das observações 26 .

Atendendo ao princípio da modelagem multinível, a análise estatística foi processada iniciando-se pelo nível hierárquico mais baixo, ou seja, o nível que contempla as relações entre as variáveis medidas no nível da criança e a determinação do déficit linear, uma vez que são es- 
tas as unidades que estão agrupadas nos demais conglomerados 27 . Nesse sentido, essas unidades estão agrupadas na unidade intermediária - segundo nível hierárquico -, ou seja, o nível dos domicílios, e estes, por sua vez, estão inseridos em conglomerados de níveis mais altos - os municípios 28.

Como estratégia analítica, inicialmente avaliou-se a importância estatística de cada nível hierárquico na explicação do crescimento linear, especificando-se a equação de regressão com base na variável dependente e naquelas que representavam cada nível da hierarquia, ou seja, criança, domicílio e município; sem incluir no modelo quaisquer das variáveis independentes, obtendo-se assim a estimativa da variabilidade atribuída aos diferentes níveis de agregação. A significância da variabilidade nos diferentes níveis de agregação foi verificada via teste de Wald. Após esse procedimento concluiu-se que os três níveis eram significantes, indicando o uso do modelo de intercepto aleatório na base de dados deste estudo.

Em seguida, realizou-se a análise de regressão univariada, testando cada variável separadamente para selecionar as possíveis variáveis que integrariam o modelo multivariado. Nessa etapa adotou-se a significância estatística de 0,20 para a entrada das variáveis no modelo; essa probabilidade foi estipulada para que possíveis preditoras do evento não fossem excluídas da análise 29 .

O pressuposto da normalidade foi avaliado pela análise de resíduo ${ }^{30}$. Assim, os resultados dessas análises atestam a não violação do pressuposto da normalidade, indicando mais uma vez a pertinência da utilização da técnica da regressão linear múltipla para explorar as associações de interesse.

Posteriormente procedeu-se a construção do modelo final de regressão linear multivariada, incluindo na análise todas as variáveis selecionadas na regressão univariada referentes à criança (primeiro nível). Nessa etapa da análise, as variáveis desse nível que conservaram significância estatística ( $p$ valor $<0,05$ ) foram mantidas no modelo. A esse modelo foram acrescentadas as variáveis do domicílio (nível 2) e nova análise foi processada, sendo mantidas no modelo as variáveis com significância estatística previamente estipulada $(\mathrm{p}<0,05)$. Em seguida, mantendo-se os mesmos procedimentos descritos para a inclusão das variáveis dos níveis 1 e 2, foram acrescentadas ao modelo as variáveis do município (terceiro nível) e nova análise foi processada. Ao final da modelagem todas as variáveis que mantiveram significância estatística $(p<0,05)$ foram consideradas como preditoras do déficit altura/ idade.
O poder explicativo do modelo foi avaliado adotando-se o procedimento de validação cruzada, calculando-se o coeficiente de correlação de Pearson entre os valores preditos pelo modelo em parte da amostra e valores observados nos demais elementos da amostra para o indicador antropométrico altura/idade.

As análises foram realizadas utilizando-se do programa MLwiN (Centre for Multilevel Modelling, Bristol, Reino Unido).

\section{Resultados}

A descrição da população investigada está apresentada nas Tabelas 1 e 2 . Observou-se que a maioria das crianças dos 15 municípios tem 24 meses de idade ou mais; a distribuição entre os sexos não mostrou tendência definida entre os municípios de ambos os estados; maiores prevalências de déficit de crescimento linear (A/I) foram observadas nos municípios baianos, alcançando mais de $10 \%$ na maioria deles; déficits menos acentuados foram identificados para o indicador antropométrico peso/idade para os municípios de ambos os estados (Tabela 1).

A escolaridade materna, avaliada segundo anos de estudo, foi maior de cinco anos para todos os municípios paulistas enquanto que, este valor só foi alcançado por quatro municípios baianos (Presidente Dutra, Salinas das Margaridas, São Félix e Santa Inês). Os municípios de São Paulo apresentaram melhores condições econômicas e ambientais conforme mostraram os dados do IDH, do índice econômico e do índice ambiental. Esses municípios apresentaram ainda condições mais favoráveis de assistência pré-natal e vacinação para as mães e seus filhos (Tabela 2).

A prevalência do baixo peso ao nascer foi similar nos 15 municípios estudados, enquanto a proporção de crianças que receberam leite materno (independentemente do tempo e regime alimentar) foi bastante variável, no entanto a prevalência de aleitamento materno foi baixa em todos os municípios. A prevalência de esquema vacinal incompleto foi mais alta em todos os municípios baianos e expressivamente mais baixa naqueles de São Paulo. Observou-se ainda alta freqüência de história de desnutrição, segundo referência materna, em todos os municípios investigados (Tabela 2)

Os resultados da análise univariada estão apresentados na Tabela 3. Para o nível 1 da hierarquia (nível da criança), observou-se associação positiva e significante com a média do indicador altura/idade: o peso ao nascer e a idade da criança na faixa etária de 24 a 36 meses. A 
Distribuição das características biológicas das crianças menores de cinco anos segundo os municípios de residência.

Estados da Bahia e de São Paulo, 2000-2001.

\begin{tabular}{|c|c|c|c|c|c|c|c|c|}
\hline \multirow[t]{2}{*}{ Municípios } & \multicolumn{4}{|c|}{ Faixa etária (meses) } & \multicolumn{2}{|c|}{ Sexo } & \multicolumn{2}{|c|}{ Estado antropométrico } \\
\hline & $0-5$ & $6-11$ & $12-23$ & $\geq 24$ & Masculino & Feminino & Altura/Idade * & Peso/Idade * \\
\hline \multicolumn{9}{|l|}{ Bahia } \\
\hline Acajutiba & 7,2 & 6,8 & 24,2 & 61,9 & 48,4 & 51,6 & 10,8 & 6,7 \\
\hline Cipó & 8,5 & 9,6 & 24,8 & 57,0 & 49,4 & 50,6 & 15,2 & 8,5 \\
\hline Gongogi & 10,8 & 10,8 & 17,1 & 61,4 & 55,1 & 44,9 & 13,9 & 12,0 \\
\hline Itiruçu & 12,7 & 6,9 & 20,7 & 59,6 & 49,9 & 50,1 & 12,8 & 9,6 \\
\hline Milagres & 9,0 & 9,7 & 22,0 & 59,2 & 49,2 & 50,8 & 11,2 & 7,5 \\
\hline Presidente Dutra & 11,4 & 11,1 & 17,5 & 59,9 & 47,8 & 52,2 & 6,1 & 3,4 \\
\hline Salinas das Margaridas & 9,9 & 7,8 & 20,1 & 62,2 & 49,0 & 51,0 & 10,5 & 12,3 \\
\hline São Félix & 10,6 & 10,6 & 18,6 & 60,2 & 53,8 & 46,2 & 8,0 & 5,8 \\
\hline Santa Inês & 7,0 & 12,2 & 21,3 & 59,6 & 50,4 & 49,6 & 10,6 & 9,5 \\
\hline Serrolândia & 8,7 & 10,0 & 22,0 & 59,3 & 53,8 & 46,2 & 8,0 & 4,7 \\
\hline \multicolumn{9}{|l|}{ São Paulo } \\
\hline Bady Bassit & 8,1 & 11,1 & 26,8 & 54,0 & 49,5 & 50,5 & 2,1 & 2,0 \\
\hline Bofete & 11,4 & 16,0 & 18,9 & 53,7 & 51,4 & 48,6 & 4,0 & 0,0 \\
\hline Jaborandir & 8,2 & 7,2 & 20,3 & 64,3 & 50,7 & 49,3 & 2,5 & 0,0 \\
\hline Morungaba & 9,6 & 9,6 & 19,1 & 61,8 & 50,6 & 49,2 & 6,9 & 2,9 \\
\hline Riversul & 9,0 & 7,1 & 18,4 & 65,5 & 48,6 & 51,4 & 9,0 & 4,7 \\
\hline
\end{tabular}

* <-2 DP.

associação negativa e significante foi observada entre a média do indicador altura/idade e o sexo feminino, internamento prévio, coriza (referida nos últimos 15 dias), esquema de vacinação incompleto, ausência do consumo do leite materno, percepção materna da saúde da criança (regular ou ruim) e história de desnutrição prévia (Tabela 3).

Para o nível 2 da hierarquia (nível do domicílio), observou-se que a média do indicador altura/idade aumentava à medida que aumentava a renda familiar (salário mínimo da época: $\mathrm{R} \$ 136,00)$, a pontuação dos índices ambiental e econômico, e o nível da escolaridade materna. O trabalho materno fora do domicílio mostrou-se associado positivamente à média do indicador altura/idade.

No nível 3 da hierarquia (nível do município), observou-se associação positiva e estatisticamente significante entre a média do indicador altura/idade com o aumento do volume de água consumido, aumento da arrecadação per capita do município, com a pontuação do índice municipal de desenvolvimento humano e com o aumento percentual do número de consultas prestadas pela assistência pré-natal do município. Identificou-se associação inversa e significante entre a média do indicador altura/idade e razão de concentração de renda segundo área dos imóveis rurais (Tabela 3 ).

Os resultados do modelo multivariado final, contemplando todas as variáveis preditoras que mantiveram significância estatística $(p<0,05)$, estão apresentados na Tabela 4 e na Figura 1. Esses resultados indicaram que no primeiro nível da hierarquia (nível da criança), o peso ao nascer associou-se positivamente com a média do indicador altura/idade. Assim, quando o peso ao nascer aumentava em um quilo aumentava também a estimativa média do indicador antropométrico altura/idade em 0,407 z scores (IC: 0,34;0,47). Associação direta e significante foi observada também com a idade da criança, particularmente para as faixas etárias de 24 a 36 meses e acima de 36 meses, com os respectivos acréscimos de 0,459 (IC: 0,$35 ; 0,56)$ e 0,174 (IC: 0,08;0,27) na média do indicador altura/idade.

Ainda para variáveis relacionadas com a criança, identificou-se que o esquema vacinal incompleto decrescia o valor médio do indicador altura/idade em 0,110 (IC: -0,21;-0,01) e a ausência do consumo do leite materno o fez em 0,198 (IC: -0,38;-0,01). Identificou-se também que a percepção materna sobre o estado de saúde da criança funcionou como preditor do indicador altura/idade. Nesse sentido, quando o estado de 
Distribuição das características maternas, do domicílio e das crianças segundo os municípios de residência. Estados da Bahia e de São Paulo, Brasil, 2000-2001.

\begin{tabular}{|c|c|c|c|c|c|c|c|c|c|}
\hline Municípios & $\begin{array}{c}\text { Escolaridade } \\
\text { materna } \\
\text { (anos) }\end{array}$ & $\begin{array}{c}\text { Índice } \\
\text { ambiental } \\
(\bar{X})\end{array}$ & $\begin{array}{c}\text { Índice } \\
\text { econômico } \\
(\bar{X})\end{array}$ & $\mathrm{IDH}$ * & $\begin{array}{l}\text { Assistência } \\
\text { pré-natal * }\end{array}$ & $\begin{array}{c}\text { Peso } \\
\text { ao nascer } \\
(\mathrm{kg}) \star \star \star\end{array}$ & $\begin{array}{c}\text { Aleitamento } \\
\text { materno } \\
(\%){ }^{\#}\end{array}$ & $\begin{array}{c}\text { Vacinação } \\
\text { incompleta } \\
\text { (\%) \#\# }\end{array}$ & $\begin{array}{l}\text { História de } \\
\text { desnutrição } \\
\text { (\%) \#\#\# }\end{array}$ \\
\hline \multicolumn{10}{|l|}{ Bahia } \\
\hline Acajutiba & 4,99 & 25,44 & 8,36 & 0,396 & 22,5 & 3,27 & 8,3 & 36,0 & 20,0 \\
\hline Cipó & 4,86 & 23,59 & 8,24 & 0,415 & 30,7 & 3,31 & 3,0 & 26,0 & 12,3 \\
\hline Gongogi & 3,82 & 22,17 & 7,38 & 0,377 & 60,2 & 3,25 & 4,8 & 31,0 & 18,4 \\
\hline Itiruçu & 4,14 & 23,61 & 8,26 & 0,428 & 19,9 & 3,31 & 4,8 & 23,0 & 21,7 \\
\hline Milagres & 4,67 & 21,91 & 7,02 & 0,447 & 18,3 & 3,35 & 2,9 & 16,0 & 13,0 \\
\hline Presidente Dutra & 5,20 & 22,39 & 7,61 & 0,394 & 69,6 & 3,38 & 1,7 & 11,0 & 23,0 \\
\hline Salinas das Margaridas & 6,14 & 27,22 & 8,05 & 0,775 & 9,63 & 3,15 & 2,7 & 20,0 & 25,0 \\
\hline São Félix & 5,95 & 22,99 & 9,21 & 0,472 & 59,0 & 3,33 & 4,0 & 41,0 & 8,0 \\
\hline Santa Inês & 5,42 & 24,38 & 7,83 & 0,391 & 59,4 & 3,38 & 6,1 & 17,0 & 20,0 \\
\hline Serrolândia & 4,95 & 22,64 & 5,76 & 0,407 & 38,4 & 3,33 & 3,7 & 14,0 & 24,0 \\
\hline \multicolumn{10}{|l|}{ São Paulo } \\
\hline Bady Bassit & 6,86 & 33,33 & 17,03 & 0,776 & 75,1 & 3,21 & 4,1 & 2,7 & 12,0 \\
\hline Bofete & 6,20 & 32,27 & 12,23 & 0,681 & 57,0 & 3,24 & 5,1 & 12,0 & 14,0 \\
\hline Jaborandir & 5,65 & 32,78 & 13,49 & 0,743 & 73,3 & 3,21 & 8,4 & 5,2 & 19,0 \\
\hline Morungaba & 6,05 & 31,58 & 15,62 & 0,763 & 17,4 & 3,21 & 13,0 & 2,2 & 14,0 \\
\hline Riversul & 5,25 & 30,80 & 10,68 & 0,537 & 63,0 & 3,17 & 9,1 & 7,7 & 19,0 \\
\hline
\end{tabular}

* Índice de Desenvolvimento Humano;

** Percentual de recém-nascidos cujas mães fizeram seis ou mais consultas no pré-natal;

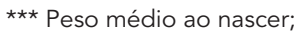

\# Proporção de crianças que receberam leite materno;

\#\# Proporção de crianças com esquema vacinal incompleto para BCG, Tríplice e Poliomielite;

\#\#\# Proporção de crianças cujas mães referiram história de desnutrição.

saúde da criança era regular ou ruim, segundo a percepção materna, o decréscimo no valor médio deste indicador era de 0,136 (IC: -0,23;-0,04). Da mesma maneira que o relato de desnutrição prévia em algum período da vida da criança implicou o decréscimo de 0,503 (IC: -0,60;-0,40) no valor médio do indicador altura/idade.

As variáveis do nível do domicílio relacionadas às condições ambientais e econômicas da família associaram-se positivamente com a adequação da altura da criança. Assim, o aumento de um ponto nos índices ambiental e econômico imprimiu, respectivamente, aumento de 0,015 (IC: 0,01;0,02) e 0,019 (IC: 0,01;0,03) no valor médio do indicador altura/idade, e o aumento de um ano de estudo na escolaridade materna expressou aumento de $0,026 \mathrm{z}$ score neste indicador (IC: 0,$01 ; 0,04)$.

Dentre as variáveis que integraram o nível do município, observou-se que para cada unidade de aumento no porcentual de 6 ou mais consultas no pré-natal promoveu aumento de 0,006 no valor médio do z score do indicador altura/idade (IC: 0,002-0,01). Identificou-se também que quando o IDH aumentava em 10\% aumentava também em 0,1009 (IC: 0,55;1,47) o valor médio do indicador altura/idade.

Os resultados da análise da variabilidade do crescimento linear atribuída a cada nível hierárquico, indicaram que a variabilidade do crescimento linear no nível do domicílio e do município foi significante (Tabela 5). Os resultados indicaram também que o modelo proposto explicou $27,4 \%$ da variabilidade do crescimento linear das crianças investigadas. Esse parâmetro foi obtido pela estimativa da diferença entre a variância do modelo sem preditores $(1,273)$ e a variância do modelo com preditores $(0,924)$; o resultado desta diferença $(0,349)$ foi dividido pela variância total do modelo sem preditores $(1,273)$ e multiplicado por 100. Da variabilidade total explicada pelo modelo, $15,7 \%$ foram atribuídas às variáveis municipais (IDH e assistência pré-natal), 33\% às variáveis domiciliares (índice ambiental, índice 
Regressão linear univariada para predição do déficit de crescimento linear, segundo níveis de hierarquia. Estados da Bahia e de São Paulo, Brasil, 2000-2001.

\begin{tabular}{|c|c|c|}
\hline Variáveis & Estimativa (SE) & IC95\% \\
\hline \multicolumn{3}{|l|}{ Nível 1: criança } \\
\hline \multicolumn{3}{|l|}{ Sexo } \\
\hline Feminino & $-0,089(0,041)$ & $-0,171 ;-0,007$ \\
\hline \multicolumn{3}{|l|}{ Idade (meses) } \\
\hline $0-6$ & - & - \\
\hline $12-24$ & $-0,165(0,053)$ & $-0,27 ;-0,06$ \\
\hline $24-36$ & $0,291(0,053)$ & 0,$18 ; 0,40$ \\
\hline Acima de 36 & $0,045(0,046)$ & $-0,05 ; 0,14$ \\
\hline Peso ao nascer (g) & $0,492(0,033)$ & 0,$43 ; 0,56$ \\
\hline \multicolumn{3}{|l|}{ Vacinação completa } \\
\hline Não & $-0,242(0,050)$ & $-0,34 ;-0,14$ \\
\hline \multicolumn{3}{|l|}{ Aleitamento Materno } \\
\hline Não & $-0,157(0,083)$ & $-0,32 ; 0,01$ \\
\hline \multicolumn{3}{|l|}{ Percepção materna da saúde da criança } \\
\hline Muito boa/Boa & - & - \\
\hline Regular/Ruim & $-0,233(0,046)$ & $-0,32 ;-0,14$ \\
\hline \multicolumn{3}{|l|}{ História prévia de desnutrição } \\
\hline $\operatorname{Sim}$ & $-0,582(0,045)$ & $-0,67 ;-0,49$ \\
\hline \multicolumn{3}{|l|}{ Internamento prévio } \\
\hline Sim & $-0,095(0,04)$ & $-0,175 ;-0,015$ \\
\hline \multicolumn{3}{|l|}{ Diarréia * } \\
\hline Sim & $-0,054(0,051)$ & $-0,156 ; 0,048$ \\
\hline \multicolumn{3}{|l|}{ Febre * } \\
\hline Sim & $-0,010(0,052)$ & $-0,114 ; 0,094$ \\
\hline \multicolumn{3}{|l|}{ Coriza * } \\
\hline Sim & $-0,125(0,046)$ & $-0,217 ;-0,033$ \\
\hline \multicolumn{3}{|l|}{ Perda de apetite* } \\
\hline Sim & $-0,026(0,048)$ & $-0,122 ; 0,07$ \\
\hline \multicolumn{3}{|l|}{ Nível 2: domicílio } \\
\hline Índice ambiental & $0,041(0,003)$ & 0,$03 ; 0,05$ \\
\hline Índice econômico & $0,049(0,004)$ & 0,$04 ; 0,06$ \\
\hline Anos de escolaridade materna & $0,066(0,006)$ & 0,$05 ; 0,08$ \\
\hline Renda familiar per capita (salário mínimo) & $0.647(0.10)$ & 0,$447 ; 0,847$ \\
\hline Trabalho materno fora de casa & $0.136(0.051)$ & 0,$034 ; 0,238$ \\
\hline \multicolumn{3}{|l|}{ Nível 3: município } \\
\hline Assistência pré-natal no município ** & $0,007(0,003)$ & 0,$001 ; 0,01$ \\
\hline Índice municipal de desenvolvimento humano (IDH) & $1,172(0,332)$ & 0,$51 ; 1,84$ \\
\hline Razão de concentração de renda & $-0,259(0,184)$ & $-0,627 ; 0,109$ \\
\hline \multicolumn{3}{|l|}{ Razão de concentração de renda segundo } \\
\hline área dos imóveis rurais & $-0,072(0,030)$ & $-0,132 ;-0,012$ \\
\hline Grau de urbanização & $0,005(0,004)$ & $-0,003 ; 0,013$ \\
\hline Volume de água consumido $\left(\mathrm{m}^{3}\right)$ & $0,0066(0,002)$ & 0,$0026 ; 0,0106$ \\
\hline Arrecadação total & $-0,0024(0,0016)$ & $-0,0056 ; 0,0008$ \\
\hline Arrecadação per capita & $0,0006(0,0002)$ & 0,$0002 ; 0,001$ \\
\hline Renda média do chefe de família (salário mínimo) & $0,0006(0,0023)$ & $-0,004 ; 0,0052$ \\
\hline Valor da produção animal & $0,00001(0,00017)$ & $-0,00033 ; 0,00035$ \\
\hline
\end{tabular}

* Nos 15 dias anteriores à entrevista;

** Percentual de recém-nascidos cujas mães fizeram seis ou mais consultas no pré-natal. 
Regressão linear multivariada para predição do déficit de crescimento linear, segundo níveis de hierarquia. Estados da Bahia e de São Paulo, Brasil, 2000-2001.

\begin{tabular}{|c|c|c|}
\hline Variáveis & Estimativa (SE) & IC95\% \\
\hline Intercepto & $-0,514(0,037)$ & \\
\hline \multicolumn{3}{|l|}{ Nível 1: criança } \\
\hline \multicolumn{3}{|l|}{ Idade (meses) } \\
\hline $0-6$ & - & - \\
\hline $12-24$ & $-0,031(0,055)$ & $-0,14 ; 0,08$ \\
\hline $24-36$ & $0,459(0,053)$ & 0,$35 ; 0,56$ \\
\hline Acima de 36 & $0,174(0,05)$ & 0,$08 ; 0,27$ \\
\hline Peso ao nascer (g) & $0,407(0,034)$ & 0,$34 ; 0,47$ \\
\hline \multicolumn{3}{|l|}{ Vacinação completa } \\
\hline Sim & - & - \\
\hline Não & $-0,110(0,053)$ & $-0,21 ;-0,01$ \\
\hline \multicolumn{3}{|l|}{ Aleitamento Materno } \\
\hline Sim & - & - \\
\hline Não & $-0,198(0,094)$ & $-0,38 ;-0,01$ \\
\hline \multicolumn{3}{|l|}{ Percepção materna da saúde da criança } \\
\hline Muito boa/Boa & - & - \\
\hline Regular/Ruim & $-0,136(0,05)$ & $-0,23 ;-0,04$ \\
\hline \multicolumn{3}{|l|}{ História prévia de desnutrição } \\
\hline Não & - & - \\
\hline Sim & $-0,503(0,051)$ & $-0,60 ;-0,40$ \\
\hline \multicolumn{3}{|l|}{ Nível 2: domicílio } \\
\hline Índice ambiental & $0,015(0,0004)$ & 0,$01 ; 0,02$ \\
\hline Índice econômico & $0,019(0,004)$ & 0,$01 ; 0,03$ \\
\hline Anos de escolaridade materna & $0,026(0,007)$ & 0,$01 ; 0,04$ \\
\hline \multicolumn{3}{|l|}{ Nível 3: município } \\
\hline Assistência pré-natal no município * & $0,006(0,002)$ & 0,$002 ; 0,01$ \\
\hline Índice municipal de desenvolvimento humano (IDH) & $1,009(0,234)$ & 0,$55 ; 1,47$ \\
\hline
\end{tabular}

* Percentual de recém-nascidos cujas mães fizeram seis ou mais consultas no pré-natal.

Estimativa da variabilidade na análise linear multinível para predição do déficit de crescimento linear. Estados da Bahia e de São Paulo, Brasil, $2000-2001$.

\begin{tabular}{|c|c|c|c|c|c|}
\hline Níveis & $\begin{array}{c}\text { Modelos sem } \\
\text { preditores } \\
\text { [variância (erro padrão var)] }\end{array}$ & $\begin{array}{c}\text { Modelo } \\
\text { completo } \\
\text { [variância (erro padrão var)] }\end{array}$ & $\begin{array}{l}\text { Diferença } \\
\text { das } \\
\text { variâncias1 }\end{array}$ & $\begin{array}{l}\text { \% variabilidade } \\
\text { atribuída } \\
\text { a cada nível * }\end{array}$ & $\begin{array}{l}\% \text { variabilidade } \\
\text { explicada pelo } \\
\text { modelo final ** }\end{array}$ \\
\hline Criança & {$[0,736(0,032)]$} & {$[0,557(0,032)]$} & 0,179 & 57,8 & 51,3 \\
\hline Domicílio & {$[0,468(0,037)]$} & {$[0,353(0,036)]$} & 0,115 & 36,8 & 33,0 \\
\hline \multirow[t]{2}{*}{ Município } & {$[0,069(0,027)]$} & {$[0,014(0,007)]$} & 0,055 & 5,42 & 15,7 \\
\hline & 1,273 & 0,924 & 0,349 & & \\
\hline
\end{tabular}

* Cálculo realizado para o modelo sem preditores, resultado obtido pela divisão da variância em cada nível pela variância total (1,273) multiplicado por 100;

** A variabilidade explicada pelo modelo é obtida pela diferença entre a variância ${ }^{1}$ em cada nível no modelo sem preditores com a variância no modelo com preditores, dividido pelo total destas diferenças $(0,349)$ e multiplicado por 100. 
econômico e escolaridade materna) e 51,3\% às variáveis relacionadas à criança (idade, peso ao nascer, vacinação, aleitamento materno, percepção materna sobre estado de saúde da criança e história prévia de desnutrição). $\mathrm{Na}$ análise do poder explicativo do modelo final encontrou-se coeficiente de correlação (r) de 0,42; mostrando um razoável poder explicativo do modelo proposto para os dados utilizados.

\section{Discussão}

Conforme hipótese inicial, os resultados deste estudo indicaram que a variabilidade do crescimento linear na infância está situada em diferentes níveis de complexidade, a exemplo das condições relacionadas aos municípios, domicílios e por fatores diretamente associados à criança. Nesse sentido, as condições estruturais da sociedade, entendidas como fontes, recursos e políti- cas públicas oferecidas pela esfera governamental, se materializam nas condições econômicas e de acesso da família aos serviços e, se comportam como importantes preditores do crescimento na infância (Figura 1). Assim, os determinantes no nível do município que definem as condições de vida, a qualidade e a quantidade dos serviços prestados à população, intermediam a relação de acesso aos bens e serviços, situados no nível do domicilio e se expressam nas condições de saúde e nutrição na infância.

Quando essas condições se manifestam como promotoras da iniqüidade social, econômica e da saúde, atuam constrangendo o potencial do crescimento pleno da criança. Nesse sentido, a limitação imposta por esses campos da determinação, identificada neste estudo, no nível do município se expressa de forma contundente no nível do domicilio e restringe a capacidade da família em prestar a adequada assistência à criança, restringindo a adoção materna de um

Figura 1

Modelo mutínivel final dos preditores do déficit de crescimento linear em crianças pré-escolares.

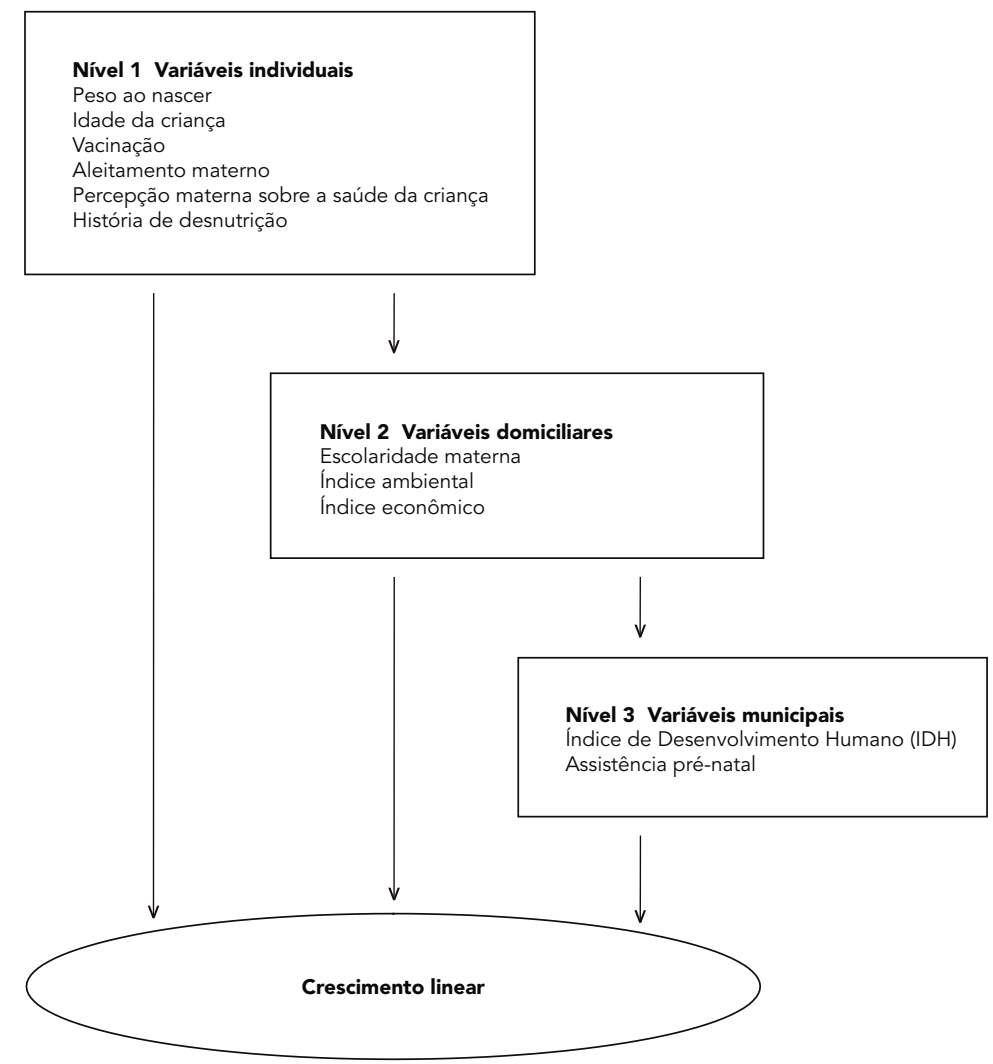


conjunto de ações que poderiam contribuir na promoção da saúde e nutrição da criança, a exemplo da vacinação, do aleitamento materno e da adoção de práticas que redundariam na melhoria do peso ao nascer. Assim, a confluência desses fatores culmina com a limitação do enfrentamento da própria criança em superar com êxito as condições adversas do ambiente social e econômico em que está inserida, para assegurar a adequada condição de saúde e nutrição.

Assim, o modelo final deste estudo mostra que o baixo peso ao nascer, o desmame precoce ou a ausência da amamentação, o esquema vacinal incompleto, ademais da história prévia de desnutrição associam-se diretamente com o déficit do indicador altura/idade.

Nota-se, que os determinantes do crescimento linear na infância se distribuem de maneira diferenciada entre os vários níveis de mediação de hierarquia, situados na esfera do ambiente social e econômico em que vivem as crianças e suas famílias. Nesse sentido, os resultados deste estudo corroboram com aqueles que identificaram que a baixa renda familiar, restrito acesso a serviços de saúde, condições precárias de saneamento básico e a baixa escolaridade materna constituem importantes constrangedores das condições de saúde e nutrição na infância $11,31,32,33$.

Cabe comentar também, que o modelo conceitual da desnutrição na infância sugerido pelo UNICEF 11 representa um passo importante na avaliação dos determinantes da desnutrição infantil. No entanto, o nosso estudo apresenta inovações por incorporar a modelagem multinível, uma abordagem relativamente recente e mais apropriada para dados com estrutura hierárquica, na explicação das variáveis contextuais e individuais relacionadas à condição sócio-econômica da família e do ambiente em que vive a criança e sua família, enquanto determinantes da adequação plena do crescimento linear. O modelo final, com razoável poder estatístico, indica a influência de um leque relevante desses fatores situados nos diferentes níveis de agregação como constrangedores do crescimento linear na infância.
Assim, os determinantes situados no nível do município explicaram em 15,7\% a variabilidade do déficit de crescimento linear das crianças investigadas, enquanto aqueles situados no nível do domicilio contribuíram com 33\% da explicação e, os fatores imediatos, ou seja, aqueles situados no nível individual ou da própria criança, explicaram 51,3\% da variabilidade observada.

É possível interpretar esses resultados como indicativos de que, melhorando as condições de vida das crianças e de suas famílias, ampliando o acesso aos bens e consumo necessários à sobrevivência, bem como melhorando as condições do contexto (município) em que a criança vive, esta pode desenvolver plenamente a sua capacidade de enfrentamento das condições biológicas e de morbidade, e responder de forma mais exitosa aos agravos do ambiente que possam comprometer o seu potencial pleno de crescimento.

Por último cabe comentar que, embora a definição do número e tipos de municípios de cada Estado incluídos neste estudo tivessem sido definidos por critérios de viabilidade logística e da disponibilidade de recursos para executar o estudo; não sendo, portanto mensurado $a$ priori o poder dos testes para o tamanho da amostra no nível do município, foi suficientemente grande para detectar pelo menos parte da variabilidade atribuída a este nível, atestada pela significância observada para as variáveis do município. Entende-se assim, que enquanto a generalização dos resultados do estudo for feita com cautela, aliás, como para a grande maioria dos estudos epidemiológicos, a sua validade interna estará preservada.

Portanto, pode-se concluir que o modelo adotado neste estudo contribuiu para o entendimento dos fatores determinantes do déficit do crescimento linear na infância, explorando as relações hierárquicas entre os diferentes determinantes, dispostos em diferentes níveis de agregação, definido assim um complexo sistema de inter-relações e, desta maneira, os seus resultados podem ser úteis para a definição de intervenções efetivas no campo da nutrição e da saúde de crianças e no monitoramento mais intensivo dos principais fatores de risco que se associam à ocorrência do déficit linear na infância. 


\section{Resumo}

Este estudo teve como objetivo identificar os determinantes do déficit de crescimento linear em crianças menores de cinco anos, utilizando modelagem multinível. Foi estudada uma amostra de 3.746 pré-escolares de 15 municípios brasileiros (dez do Estado da Bahia e cinco do Estado de São Paulo), construída em três estágios (municípios, domicílios e crianças). Os dados municipais foram obtidos do censo demográfico de 1991. Os dados referentes aos indivíduos e domicílios foram coletados entre 1999 e 2001, constando de questionários estruturados e medições de peso e altura das crianças. Para análise utilizou-se o programa MLwiN. No modelo multinivel final observou-se que baixo IDH e inadequada assistência pré-natal, no nível dos municípios; baixas condições ambientais, econômicas e da escolaridade materna, no nível dos domicílios; o baixo peso ao nascer, esquema vacinal incompleto, história prévia de desnutrição e ausência do aleitamento ao peito, no nível das crianças, mostraram-se fortemente associados ao déficit do crescimento linear das crianças estudadas. A variabilidade total da desnutrição explicada pelo modelo foi de 27,4\%, desta, $51,3 \%, 33 \%$ e 15,7\% foram explicadas, respectivamente, pelas variáveis dos níveis da criança, do domicílio e do município.

Crescimento e Desenvolvimento; Desnutrição; Pré-Escolar

\section{Colaboradores}

N. F. Oliveira e A. C. R. Braga-Junior participaram da elaboração, implementação e interpretação da análise estatística. L. P. M. Oliveira, A. M. O. Assis, M. L. Barreto, S. R. D. M. Saldiva e M. M. L. Escuder coordenaram o trabalho de campo, participaram da elaboração de instrumento para coleta de dados e realizaram esta atividade. L. P. M. Oliveira, A. M. O. Assis e M. L. Barreto participaram da elaboração final do artigo. L. P. M. Oliveira, A. M. O. Assis, M. L. Barreto, M. F. F. P. Nunes, M. H. D'A. Benício e S. I. Venâncio realizaram a implementação e interpretação dos resultados e revisão final do artigo.

\section{Referências}

1. Monteiro CA, Conde WL. Tendência secular da desnutrição e da obesidade na infância na Cidade de São Paulo (1974-1996). Rev Saúde Publica 2000; 34(6 Suppl):52-61.

2. De Onis M, Frongillo EA, Blosser M. Is malnutrition declining? An analysis of changes in levels of child malnutrition since 1980. Bull World Health Organ 2000; 78:1222-33.

3. World Health Organization. Reducing risks, promoting health life. Supporting material for the Word Health Report 2002. Geneva: World Health Organization; 2002.

4. Schroeder DG, Brown KB. Nutritional status as a predictor of child survival: summarizing the association and quantifying its global impact. Bull World Health Organ 1994; 72:569-79.
5. World Health Organization. Global database on child grow and malnutrition. Program of Nutrition Family and Reproductive Health. Geneva: World Health Organization; 1997.

6. Organização Pan-Americana da Saúde/Organização Mundial da Saúde. A saúde no Brasil. Brasília: Organização Pan-Americana da Saúde/Organização Mundial da Saúde; 1998.

7. Sociedade Civil Bem-Estar Familiar no Brasil/Programa de Pesquisa de Demografia e Saúde. Pesquisa nacional sobre demografia e saúde 1996. 2a Ed. Rio de Janeiro: Sociedade Civil Bem-Estar Familiar no Brasil/Programa de Pesquisa de Demografia e Saúde; 1999. 
8. Departamento de Atenção Básica, Secretaria de Atenção à Saúde, Ministério da Saúde. Avaliação do programa bolsa-alimentação: primeira fase. Brasília: Ministério da Saúde; 2004.

9. Duarte EC, Schneider MC, Paes-Souza R, Ramalho WM, Sardinha LMV, Silva Júnior JB, et al. Epidemiologia das desigualdades em saúde no Brasil. Um estudo exploratório. http://www.funasa.gov. $\mathrm{Br} / \mathrm{pub} / \mathrm{epi}$ _desigualdades (acessado em 22/ Jun/2003).

10. Hakim P, Solimano G. Nutrição e desenvolvimento nacional: estabelecendo a conexão. In: Valente FLS, organizador. Fome e desnutrição. Determinantes sociais. São Paulo: Cortez Editora; 1986. p. 29-37.

11. Fundo das Nações Unidas para Infância. Situação mundial da infância. Brasília: Fundo das Nações Unidas para Infância; 1998.

12. Zere E, McIntyre D. Inequities in under-five child malnutrition in South Africa. Int J Equity Health 2003; $2: 1-10$.

13. Administrative Committee on Coordination/SubCommittee on Nutrition. Third report on the world nutrition situation. Geneva: World Health Organization; 1997.

14. Diez-Roux AV. Multilevel analysis in public health research. Annu Rev Public Health 2000; 21:171-92.

15. Instituto Brasileiro de Geografia e Estatística. Censo demográfico 1991: resultado do universo relativo às características da população e dos domicílios, Bahia, $n^{\circ}$. 17. Rio de Janeiro: Instituto Brasileiro de Geografia e Estatística; 1994.

16. Benício MHD’A, Monteiro CA. Desnutrição infantil nos municípios brasileiros - risco de ocorrência. Brasília: Núcleo de Estudos Epidemiológicos em Nutrição e Saúde, Universidade de São Paulo/Fundo das Nações Unidas para Infância; 1997.

17. Barreto ML, Assis AMO, Braga-Junior ACR, Teles CAS, Monteiro CA, Mercês CF, et al. Indicadores socioeconômicos no diagnóstico e planejamento em saúde e nutrição. Salvador: Financiadora de Estudos e Projeto; 2002.

18. Fundação Sistema Estadual de Análise de Dados. Informações dos municípios paulistas: Índice Paulista de Responsabilidade Social (IPRS, 1997). http://www.seade.gov.br/cgi-bin/lingcv98/spd_ 01.ksh (acessado em 29/Ago/2005).

19. Organización Mundial de la Salud. Medición del cambio del estado nutricional. Geneva: Organización Mundial de la Salud; 1983.
20. National Center For Health Statistics. NCHS growth curves for children birth-18 years. Washington DC: US Department of Health, Education and Welfare; 1977. (Vital and Health Statistics, Series 11).

21. Issler RM, Giugliani ERJ. Identificação de grupos mais vulneráveis à desnutrição infantil pela medição do nível de pobreza. J Pediatr (Rio J) 1997; 73:101-5.

22. Associação Brasileira de Empresas de Pesquisa. Critério de classificação econômica Brasil. http:// www.anep.org.br/default.aspx (acessado em 18/ Jul/2005).

23. Goldstein H. Multilevel statistical models. $2^{\text {nd }}$ Ed. London: Edward Arnold; 1995.

24. Hox JJ. Applied multilevel analysis. Amsterdam: TT-Publikaties; 1995.

25. Yang M, Rasbash J, Goldstein H, Barbosa M. MLwiN macros for advanced multilevel modelling. London: Institute of Education; 1999.

26. Kreft IGG, De Leeuw J. Introducing multilevel modeling. Thousand Oaks: Sage Publications; 1998.

27. Dickinson LM, Basu A. Multilevel modeling and practice-based research. Ann Fam Med 2005; 3 Suppl 1:S52-60.

28. Pinheiro SMC. Modelo linear hierárquico: um método alternativo para análise de desempenho escolar [Dissertação de Mestrado]. Recife: Departamento de Estatística, Universidade Federal de Pernambuco; 2005.

29. Hosmer DW, Lemeshow S. Applied logistic regression. New York: Wiley/Interscience; 1989.

30. Armitage P, Berry G. Estadística para la investigación biomédica. 3a Ed. Madrid: Harcourt Brace; 1997.

31. Engstrom EM, Anjos LA. Déficit estatural nas crianças brasileiras: relação com condições sócio-ambientais e estado nutricional materno. Cad Saúde Pública 1999; 15:559-67.

32. Grilo LP, Carvalho LR, Silva AC, Verreschi ITN, Sawaya AL. Influência das condições socioeconômicas nas alterações nutricionais e na taxa de metabolismo de repouso em crianças escolares moradoras em favelas no Município de São Paulo. Rev Assoc Méd Bras 2000; 46:7-14.

33. Drachler ML, Andersson MCS, Leite JCC, Marshall T, Aerts DRGC, Freitas PF, et al. Desigualdade social e outros determinantes da altura em crianças: uma análise multinível. Cad Saúde Pública 2003; 19:1815-25.

Recebido em 13/Dez/2004

Versão final reapresentada em 08/Mai/2006

Aprovado em 13/Nov/2006 